\title{
Plaque complement activation and cognitive loss in Alzheimer's
} disease

\author{
David A Loeffler*1, Dianne M Camp ${ }^{1}$ and David A Bennett ${ }^{2,3}$
}

Address: ${ }^{1}$ Neurology Research Laboratory, William Beaumont Hospital Research Institute, Royal Oak, MI 48073, USA, ${ }^{2}$ Rush Alzheimer's Disease Center, Chicago, IL 60612, USA and '3 Department of Neurological Sciences, Rush University Medical Center, Chicago, IL 60612, USA

Email: David A Loeffler* - DLoeffler@beaumont.edu; Dianne M Camp - DCamp@beaumont.edu; David A Bennett - dbennett@rush.edu

* Corresponding author

Published: II March 2008

Journal of Neuroinflammation 2008, 5:9 doi:10.1 186/1742-2094-5-9

This article is available from: http://www.jneuroinflammation.com/content/5/I/9

(c) 2008 Loeffler et al; licensee BioMed Central Ltd.

This is an Open Access article distributed under the terms of the Creative Commons Attribution License (http://creativecommons.org/licenses/by/2.0), which permits unrestricted use, distribution, and reproduction in any medium, provided the original work is properly cited.
Received: 19 November 2007

Accepted: II March 2008

\begin{abstract}
Background: Complement activation is increased in Alzheimer's disease (AD), but its significance is unclear. The objective of this study was to determine the relationship between complement activation and cognition during the development of $A D$.
\end{abstract}

Methods: iC3b, C9, Bielschowsky, and Gallyas staining was performed on aged normal $(n=17)$, mild cognitively impaired $(n=12)$, and $A D(n=17-18)$ inferior temporal gyrus specimens. Plaques were counted in $10 \times$ fields with high numbers of Bielschowsky-stained plaques. One-way ANOVA was used to determine between-group differences for plaque counts and measures of cognitive function, and linear regression was used to evaluate global cognition as a function of Bielschowskystained plaques. Terms for iC3b-and C9-stained plaques were then added sequentially as additional predictors in a "mediation analysis" model.

Results: Complement was detected on plaques in all groups, and on neurofibrillary tangles only in AD specimens. iC3b, C9, and Bielschowsky-stained plaque counts increased 2.5- to 3-fold in AD vs. other groups (all $p \leq 0.01$ ). C9 staining was present on some diffuse plaques, as well as on neuritic plaques. Bielschowsky-stained and complement-stained plaque counts were highly correlated, and were negatively correlated with cognitive measures. When the Bielschowsky plaque count was used as a predictor, its correlations with cognitive measures were statistically significant, but when $\mathrm{iC} 3 \mathrm{~b}$ and $\mathrm{C} 9$ plaque counts were added as additional predictors, these correlations were no longer significant. This loss of significance was attributed to multicollinearity, i.e., high correlations between Bielschowsky-stained and complement-stained plaque counts.

Conclusion: Both early-stage (iC3b) and late-stage (C9) complement activation occurs on neocortical plaques in subjects across the cognitive spectrum; contrary to previous reports, $\mathrm{C} 9$ is present on some diffuse plaques. Because of high correlations between complement-stained and Bielschowsky-stained plaque counts, quantitative assessment of the extent to which complement activation may mediate the relationship between plaques and cognitive function could not be performed. Additional studies with animal models of $A D$ (if late-stage complement activation can be demonstrated), or possibly a trial in $A D$ patients with an inhibitor of late-stage complement activation, may be necessary to determine the significance of this process in $A D$. 


\section{Background}

Complement activation is a major inflammatory process which assists in the removal of microorganisms and cellular debris and the processing of immune complexes. Complement immunoreactivity is present in the Alzheimer's disease (AD) brain on amyloid-beta $(A \beta)$ - containing plaques, neurofibrillary tangles (NFTs), neuropil threads, and dystrophic neurites [1-4]. Activation proteins generated early in this process function as opsonins, chemokines, and anaphylatoxins [5], some of which have neuroprotective effects [6-8]. Complete complement activation by any of the three complement pathways (classical, alternative, or lectin-mediated) produces C5b-9, the membrane attack complex (MAC), which is neurotoxic [9]. The significance of this process in $\mathrm{AD}$ is unclear. Because of the presence of the MAC on plaques and NFTs, it has been suggested that complement activation may contribute to the development of $\mathrm{AD}$ [10-12]; the finding that it enhances $A \beta$ toxicity [13] supports this hypothesis. However, conflicting results with regard to this issue have been obtained from animal studies [14,15]. The MAC has not been reported on plaques in the transgenic mouse model of AD used in these studies, the amyloid precursor protein (APP) mouse [16], possibly due to suboptimal binding of mouse $\mathrm{C} 1 \mathrm{q}$ to the human $\mathrm{A} \beta$ in the plaques of these transgenic mice [17] and/or lack of appropriate antisera for detecting the mouse MAC.

In contrast to late-stage $\mathrm{AD}$, few studies have examined complement activation during the development of the disease. Lue et al. [18] reported a slight increase in plaqueassociated MAC staining in the superior frontal gyrus in "high pathology controls," non-demented elderly subjects with extensive AD-type neuropathology. This increase was markedly less than in AD patients although plaque numbers were similar between the two groups. More recently Zanjani et al. [4] found that early complement activation (C4d) on plaques in the temporal cortex increased in parallel with total plaque numbers from very mild to severe clinical $\mathrm{AD}$, whereas the MAC was detected on plaques and NFTs in some very mild cases but consistently only in severe AD. MAC staining on plaques was not observed in non-demented elderly controls. While these reports suggest that early complement activation may increase in the brain during the development of $\mathrm{AD}$, the contribution of this process to the cognitive impairment which characterizes the disease remains unknown. The objective of this investigation was therefore to evaluate the relationship of complement activation to cognition during the development of $\mathrm{AD}$. Inferior temporal gyrus specimens were examined. This region plays a major role in visual perception and recognition [19], and the information it encodes is likely to be involved in other cognitive functions including short- and long-term memory [20]. Atrophy and cholinergic deficits develop here early in the course of AD $[21,22]$.

\section{Methods \\ Brain specimens}

Histological sections ( $6 \mu \mathrm{m}$ thickness, mounted on Fisherbrand Superfrost/Plus slides, Fisher Scientific, Pittsburgh, PA) of $4 \%$ paraformaldehyde-fixed, paraffinembedded inferior temporal gyrus (Brodman area 20) were obtained from post-mortem brain specimens from non-cognitively impaired, aged normal (AN) subjects ( $\mathrm{n}=$ 17 ), individuals with mild cognitive impairment (MCI; $\mathrm{n}$ = 12), and subjects with $\mathrm{AD}(\mathrm{n}=17-18)$. These groups were based upon clinical diagnosis. Summary statistics (age, educational level, post-mortem interval [PMI]), and global neuropathology score) and measures of cognitive functioning for these subjects are shown in Tables 1 and 2 , respectively. The brain specimens were provided by the Rush Alzheimer's Disease Center (hereafter, Rush ADC), Chicago, IL, and were from individuals who had participated in the Religious Orders Study. Details of this study, which was approved by the Human Investigations Committee of Rush University Medical Center, have been published previously [23]. Subjects signed an informed consent and participated in an annual clinical evaluation including medical history, neurological examination, and a battery of cognitive performance tests. These tests assessed a range of clinical abilities commonly affected by aging and AD, including episodic memory, semantic memory, working memory, perceptual speed, and visuospatial ability. Summary measures of each type of cognitive ability were constructed for use in analyses as reported previously [24]. A global measure of cognitive function, based on all individual tests, was also constructed [25].

Table I: Subject summary statistics

\begin{tabular}{lccccc}
\hline Group & $\underline{\mathrm{n}}$ & age $(\mathrm{yrs})$ & education $(\mathrm{yrs})$ & $\underline{\text { PMI (hrs) }}$ & Global neuropathology score \\
\hline $\mathrm{AN}$ & 17 & $79.91 \pm 1.52$ & $19.41 \pm 0.94$ & $7.48 \pm 1.08$ & $0.33 \pm 0.09$ \\
$\mathrm{MCl}$ & 12 & $86.7 I^{\mathrm{a}} \pm 1.93$ & $18.83 \pm 1.18$ & $7.67 \pm 2.54$ & $0.39 \pm 0.12$ \\
$\mathrm{AD}$ & $17-18$ & $89.35^{\mathrm{a}} \pm 1.77$ & $17.78 \pm 0.98$ & $7.66 \pm 1.27$ & $1.02^{\mathrm{b}} \pm 0.13$
\end{tabular}

$A D$ and $\mathrm{MCl}$ subjects were older than $\mathrm{AN}$ subjects, but no significant differences were present between groups for educational level or PMI. The global neuropathology scores of the $A D$ group differed from $A N$ and $M C l$ subjects. Data are expressed as means $\pm S E M$. Abbreviations: $A D$, Alzheimer's disease; AN, aged normal subjects; $\mathrm{MCl}$, subjects with mild cognitive impairment; PMI, post-mortem interval. (ap $<0.05$ vs. AN; bp $<$ 0.05 vs. AN and $\mathrm{MCl}$; *data were available from $\mathrm{AD}$ specimens for $18 \mathrm{C}$-stained sections but only $17 \mathrm{iC} 3 \mathrm{~b}$-stained sections.) 
Table 2: Subject cognitive measures

\begin{tabular}{lccccccc}
\hline Group & Last MMSE & Global cognition & Episodic memory & Semantic memory & Working memory & Perceptual speed & Visuospatial ability \\
\hline AN & $27.76 \pm 0.47$ & $0.12 \pm 0.05$ & $0.28 \pm 0.08$ & $0.21 \pm 0.12$ & $-0.07 \pm 0.08$ & $-0.10 \pm 0.13$ & $0.03 \pm 0.12$ \\
MCI & $27.42 \pm 0.81$ & $-0.37 \pm 0.16$ & $-0.39 \pm 0.21$ & $-0.37 \pm 0.21$ & $0.01 \pm 0.18$ & $-0.72 \pm 0.26$ & $-0.56 \pm 0.22$ \\
AD & $16.89^{\mathrm{a}} \pm 1.99$ & $-1.7 I^{\mathrm{a}} \pm 0.21$ & $-2.19^{\mathrm{a}} \pm 0.27$ & $-1.44^{\mathrm{a}} \pm 0.24$ & $-1.24^{\mathrm{a}} \pm 0.22$ & $-1.73^{\mathrm{a}} \pm 0.22$ & $-1.16^{\mathrm{b}} \pm 0.20$ \\
\hline
\end{tabular}

$A D$ differed from the other groups with regard to last MMSE score, global cognition, episodic memory, semantic memory, working memory, and perceptual speed. Visuospatial ability was different only between $A D$ and $A N$ subjects. Data are expressed as means $\pm S E M$. Abbreviations: AD, Alzheimer's disease; AN, aged normal subjects; $\mathrm{MCl}$, subjects with mild cognitive impairment; MMSE, minimental state examination. (a $<<0.05$ vs. $\mathrm{AN}$ and $\mathrm{MCl}$; bp $<0.05$ vs. AN)

Based upon their annual evaluation, participants were classified with respect to AD and other common conditions with the potential to impact upon cognitive function. Annual follow-up evaluations were performed in a similar manner by examiners blinded to previously collected data. At the time of death, the available clinical data were reviewed and an opinion was rendered as to the most likely clinical diagnosis. Modified Bielschowsky staining [26] was performed at the Rush ADC and diffuse plaques, neuritic plaques, and NFTs were counted by a board-certified neuropathologist or trained technician as previously described [24]. Neuropathological ratings (Braak, CERAD, and NIA/Reagan scores) were then assigned by the neuropathologist. For statistical analyses, a global pathological score was constructed as described previously [24].

\section{Staining procedures}

Modified Bielschowsky staining [26], Gallyas staining [27], and immunocytochemical staining for iC3b (earlystage complement activation) and C9 (indicative of C5b9 and representing late-stage complement activation) were performed on serial sections in the Neurology Research Laboratory at William Beaumont Hospital, Royal Oak, MI. Multiple C9 molecules are present in C5b9 , and its presence can be demonstrated by staining for either C9 or C5b-9 [3]; because our preliminary studies revealed staining of higher numbers of plaques in $\mathrm{AD}$ specimens with mouse anti-iC3b and goat anti-C9 than with mouse anti-C4d and mouse anti-SC5b-9 (all from Quidel Corp., San Diego, CA), the antibodies to iC3b and C9 were used in subsequent experiments.

\section{Immunocytochemical staining for iC3b and C9}

Procedures were similar to those reported previously [28], with slight modifications. Paraformaldehyde-fixed, paraffin-embedded, Superfrost/Plus-mounted sections were heated for $1 \mathrm{hr}$ at $56^{\circ} \mathrm{C}$. The sections were subsequently deparaffinized and rehydrated through graded ethanol baths, then rinsed in Tris buffered saline (TBS; $0.1 \mathrm{M}$ Tris, $0.85 \% \mathrm{NaCl}, \mathrm{pH} 7.6$ ). (This and all subsequent rinses were performed three times at five min intervals.) They were treated for $4 \mathrm{~min}$ with $88 \%$ formic acid (Mallinkrodt Chemicals, Phillipsburg, NJ), then boiled for $30 \mathrm{~min}$ in citrate buffer, pH 6.0 (Antigen Unmasking Solution, Vector Laboratories, Burlingame, CA) and kept in the same buffer for an additional 20 min while they cooled. After rinsing in tap water and then in TBS, the sections were treated with $3 \% \mathrm{H}_{2} \mathrm{O}_{2} / 10 \%$ methanol in TBS for $30 \mathrm{~min}$ to eliminate endogenous peroxidase activity, rinsed in TBS with $0.1 \%$ Triton X-100 (hereafter, TBS-T), then treated with TBS with $0.4 \%$ Triton, $1 \%$ bovine serum albumin (BSA), and 20\% normal horse serum (NHS; Vector) for $30 \mathrm{~min}$. The specimens were then incubated overnight at $4^{\circ} \mathrm{C}$ with mouse monoclonal anti-human iC3b (Quidel; 1:100 dilution in TBS-0.2\% Triton-1\% BSA-2\% NHS) or goat anti-human C9 (Quidel; 1:5000 dilution in the same buffer). Negative controls, performed for each specimen, consisted of substituting mouse myeloma MOPC-21 (mouse IgG1-kappa of unknown antigenic specificity; Sigma-Aldrich, St. Louis, MO; 1:86 dilution) and normal goat serum (NGS; Vector; 1:5000 dilution) for anti-iC3b and anti-C9 antisera, respectively. After rinsing the sections in TBS-T, biotinylated horse anti-mouse IgG (for iC3b staining) or biotinylated horse anti-goat IgG (for C9 staining) (both from Vector; 1:200 dilution in TBS-T-BSA) was applied at room temperature for $90 \mathrm{~min}$, followed by rinsing in TBS and then incubation in avidinbiotin-horseradish peroxidase conjugate (ABC reagent, Vector; 1:100 dilution in TBS-BSA) for $1 \mathrm{hr}$. After washing in TBS, the sections were developed with 3,3'-diaminobenzidine $(\mathrm{DAB}) / \mathrm{H}_{2} \mathrm{O}_{2}$ with nickel enhancement (DAB Peroxidase Substrate Kit, Vector). iC3b- and MOPC21-stained sections were developed for $10 \mathrm{~min}$, and C9and NGS-stained sections were developed for $20 \mathrm{~min}$. Sections were then dehydrated in ethanol baths to xylene and coverslipped with Cytoseal-60 Mounting Medium (Richard-Allan Scientific, Kalamazoo, MI).

\section{Quantitation of Bielschowsky-stained and complement- stained plaques}

In Bielschowsky-stained sections, images from eight $10 \times$ objective fields from areas of high plaque densities were captured with a Nikon Eclipse 80i microscope equipped with a Nikon Digital DXM1200 camera. Nikon ACT-1 (version 2) image analysis software (Nikon USA, Melville, $\mathrm{NY}$ ) was used to find the same fields in iC3b- and C9stained serial sections, and additional images were then 
captured. The numbers of plaques in each of these images (all Bielschowsky images, followed by all iC3b and then all C9 images) were subsequently counted by one investigator (DAL) in a blinded fashion.

\section{Assessment of neuronal complement staining}

Neuronal immunoreactivity in iC3b- and C9-stained sections was scored, in a blinded fashion, on a scale from 0 to 3 , with $0=$ no complement immunoreactivity, $1=$ slight complement immunoreactivity, 2 = moderate complement immunoreactivity, and 3 = extensive complement immunoreactivity. Two observers (DAL and DMC) independently scored the specimens, and then the results were compared. When specimen scores differed (less than $10 \%$ of the specimens) the specimens were re-evaluated and a consensus was reached as to the appropriate score.

\section{NFT complement staining}

Blinded observation of the Bielschowsky- and Gallyasstained sections suggested that few or no NFTs were present in many of the specimens. This was confirmed by NFT counts that were subsequently obtained from the Rush ADC (means \pm SEM for NFT counts $/ \mathrm{mm}^{2}$ : AN, 1.35 $\pm 0.89 ; \mathrm{MCI}, 1.00 \pm 0.46 ; \mathrm{AD}, 9.39 \pm 2.93)$. Evaluation of NFT complement staining was therefore limited to specimens with NFT counts $\geq 3 / \mathrm{mm}^{2}$ (three AN, one MCI, and $12 \mathrm{AD}$ specimens). These sections were scanned with the $40 \times$ objective by an observer blinded to the diagnosis for each specimen, and a description of NFT complement staining was recorded for each.

\section{Statistical procedures}

One-way analysis of variance (ANOVA) was employed, controlling for subject age, gender, and education, to determine statistical significance between groups for Bielschowsky, iC3b, and C9 plaque counts, global neuropathology, global cognition, and the summary measures for each of the five cognitive areas referred to earlier. Post-hoc testing was performed, when necessary, via Tukey's HSD. Kruskall-Wallace (non-parametric) ANOVA was used to evaluate between-group differences for numbers of iC3b- and C9-stained neurons. Spearman rank correlations were performed to determine the strength of association between each of these variables. For plaque analyses, mean plaque counts from eight $10 \times$ fields per specimen were used; a preliminary analysis confirmed the legitimacy of including mean plaque counts, rather than counts from individual fields, in the analysis. Linear regression was employed to model global cognition as a function of global pathology and of Bielschowsky-, iC3b, and C9-stained plaques, using these terms as individual predictors and global cognition as the outcome. Terms for iC3b-stained plaques, C9-stained plaques, and the combination of $\mathrm{iC3b}$ - and C9-stained plaques were then added sequentially as additional predictors in a "mediation anal- ysis" model to determine if they would influence the strength of association between Bielschowsky plaque counts and global cognition. The analysis was subsequently repeated using each of the measures of cognitive function (episodic memory, semantic memory, working memory, perceptual speed, and visuospatial ability) as the outcome. These regression analyses were also adjusted for subject age, gender, and educational level. The significance level for all analyses was set at $p<0.05$.

\section{Results}

Age, educational level, PMI, global neuropathology score, and measures of cognitive functioning (Tables $I$ and 2)

$\mathrm{AD}$ and MCI subjects were older than AN subjects, but no significant differences were present between groups for educational level or PMI. The global neuropathology scores of the AD group differed from AN and MCI subjects, but were similar between the latter two groups. AD subjects differed from the other groups with regard to last MMSE score, global cognition, episodic memory, semantic memory, working memory, and perceptual speed. Visuospatial ability was different only between AD and AN subjects.

\section{Plaque staining (Figs. I, 2, 3)}

Complement staining was detected on plaques, neurons, glia, blood vessels, and, in $\mathrm{AD}$ specimens, occasional NFTs (Fig. 1). Bielschowsky, iC3b, and C9 plaque counts were 2.5- to 3-fold higher in $\mathrm{AD}$ specimens than in the
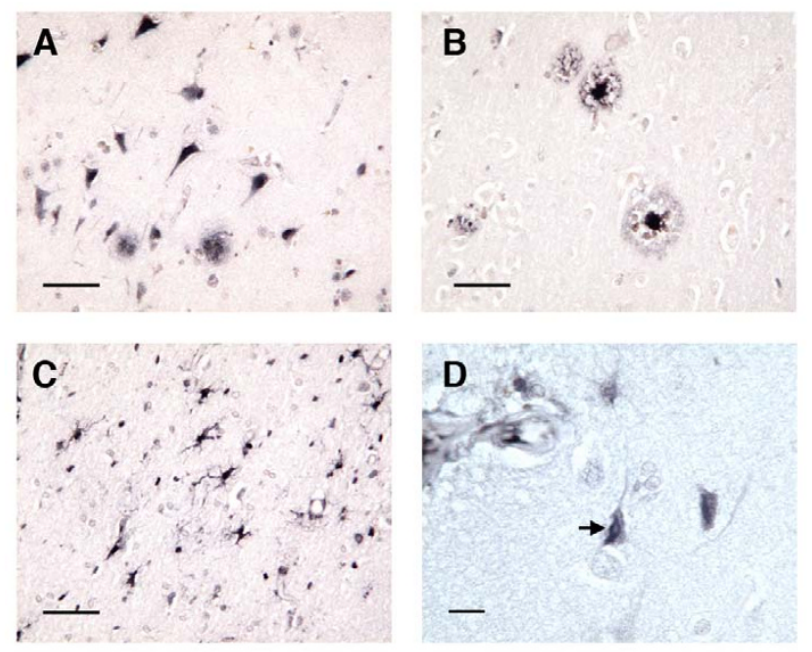

\section{Figure I}

Complement immunoreactivity in plaques, neurons, and neurofibrillary tangles. Fig. IA: iC 3 b-stained plaques and neurons, $\mathrm{MCl}$ specimen; Fig. IB: $\mathrm{C}$-stained plaques, $\mathrm{AD}$ specimen; Fig. IC: iC3b-stained glial cells, AN specimen; Fig. ID, iC3b staining of possible NFT, AD specimen (Figs. IA IC, bar $=50 \mu \mathrm{m}$; Fig. ID, bar $=12.5 \mu \mathrm{m})$. 

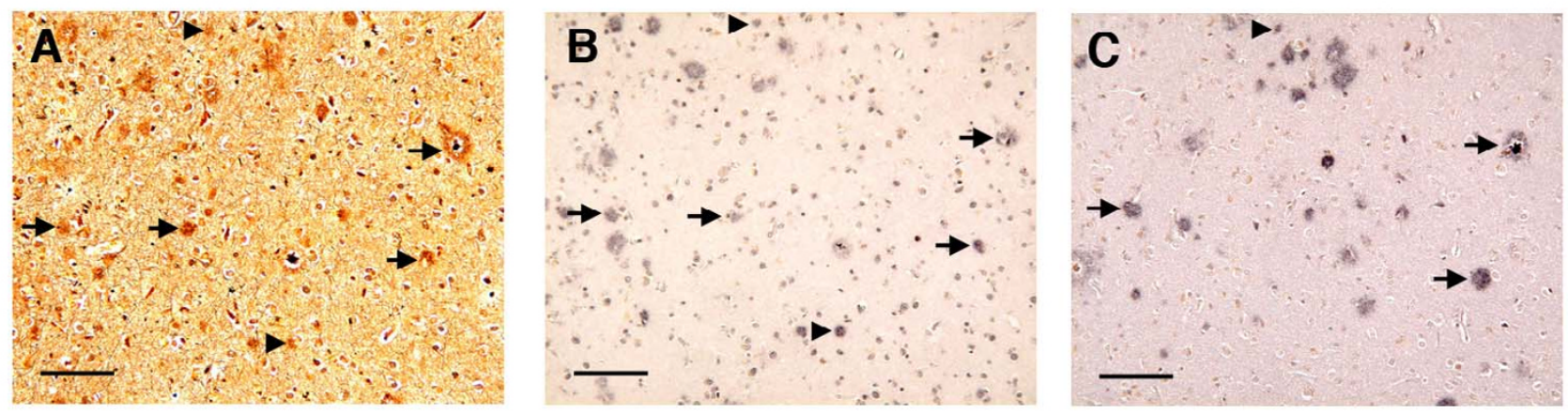

Figure 3

Alignment of plaques in Bielschowsky, iC3b, and C9-stained sections. Arrows show neuritic plaques in a Bielschowsky-stained AD specimen (Fig. 3A) that aligned with iC3b (Fig. 3B) and/or C9 plaques (Fig. 3C) in serial sections; arrowheads show diffuse plaques that aligned in the same sections (bars $=100 \mu \mathrm{m}$ ).

other groups $(\mathrm{p}=0.0002,0.0116$, and 0.0130 for Bielschowsky, iC3b, and C9 staining, respectively) (Fig. 2), but were similar between AN and MCI specimens. The numbers of Bielschowsky, iC3b, and C9-stained plaques were highly correlated (pooled specimens from all groups [Spearman rank correlations]: Bielschowsky vs. iC3b, $\mathrm{r}=$ 0.88; Bielschowsky vs. C9, r = 0.88; iC3b vs. C9, r = 0.95; all $\mathrm{p}<0.0001$ ). Assessment of plaque morphology (diffuse vs. neuritic) in Bielschowsky-stained plaques which precisely aligned with complement-stained plaques in serial sections indicated that C9 immunoreactivity was occasionally present on diffuse plaques, as well as on neuritic plaques, in all three groups of specimens (Fig. 3).

\section{Correlations between plaque staining, cognitive function, and global neuropathology (pooled data)}

Bielschowsky, iC3b, and C9 plaque counts were moderately inversely correlated with global cognition and with the five measures of cognitive ability ( $r=-0.34$ to -0.55 ; all $p<0.02$ ). All three types of plaque counts were highly correlated with global neuropathology $(\mathrm{r}=0.80$ to 0.89 ; all $p$ $<0.0001)$.

\section{Influence of Bielschowsky-, iC3b-, and C9-stained plaques on cognition}

When Bielschowsky plaques, iC3b plaques, C9 plaques, and global neuropathology were used as individual predictors, statistical significance was present vs. global cognition, episodic memory, semantic memory, working memory, and visuospatial ability as outcomes. These predictors, with the exception of global pathology $(p=$ $0.0896)$, were also significant vs. perceptual speed.

When terms for iC3b-stained plaques, C9-stained plaques, and the combination of iC3b- and C9-stained plaques were sequentially added as additional predictors to the regression analysis model examining the association of Bielschowsky-stained plaques with cognition (using global cognition and each of the five cognitive measures as outcomes), the p-values for Bielschowskystained plaques and for each of the additional predictors were no longer significant. The one exception to this was episodic memory; when a term for C9-stained plaques was added as an additional predictor, the association between Bielschowsky-stained plaques and episodic memory remained significant $(p=0.0258)$, though far less than when Bielschowsky-stained plaques were used as the only predictor ( $p=0.0004)$. The loss of statistical significance for the association of Bielschowsky-stained plaques with cognition, when iC3b- and C9-stained plaque counts were added as additional predictors, was attributed to multicollinearity. Multicollinearity exists, and is irrespective of sample size, when predictor variables are highly correlated with each other [29]; in this case, the high correlations between Bielschowsky and complement plaque counts interfered with the analysis.

\section{iC3b and C9 NFT and neuronal staining}

NFT complement staining was examined in the three AN, one MCI, and $12 \mathrm{AD}$ specimens with NFT counts $\geq 3$ / $\mathrm{mm}^{2}$. Complement immunoreactivity on NFTs was observed only in AD specimens, within apparently intact neurons (Fig. 1D). In contrast, complement staining on neurons (Fig. 1A) was detected in specimens in each of the groups. The extent of this staining was similar between groups, and it was poorly correlated with measures of cognition and with global neuropathology. The numbers of iC3b-stained and C9-stained neurons were highly correlated ( $\mathrm{r}=0.86$ for pooled data). 


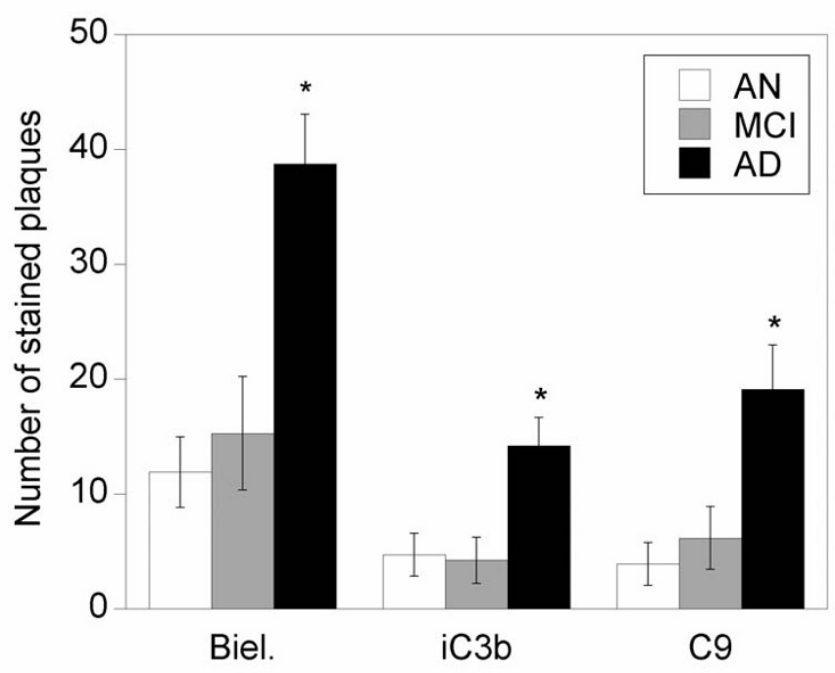

Figure 2

Bielschowsky, iC3b, and C9 plaque staining. Means for Bielschowsky, iC3b, and C9 plaque counts in eight I0x fields of high plaque density were significantly increased in $A D$ specimens vs. other groups. Data are expressed as means \pm SEM. Abbreviations: AD, Alzheimer's disease; AN, aged normal; Biel, Bielschowsky staining; $\mathrm{MCl}$, mild cognitive impairment. $\left({ }^{*} p<0.05\right.$ vs. $\mathrm{AN}$ and $\left.\mathrm{MCl}\right)$.

\section{Discussion}

Complement activation and plaque formation are mutually promoting mechanisms. Aggregated $A \beta$ efficiently binds $\mathrm{C} 1 \mathrm{q}$, activating the classical complement pathway [10], and this process further enhances $A \beta$ aggregation and fibril formation [30]. Plaque-associated complement activation has been suggested to be neurotoxic via bystander lysis [31] and also, indirectly, by chemotactic attraction of microglia [32], which when activated can secrete toxic reactive oxygen species (ROS) [33]. However, because aggregated $A \beta$ alone can also activate microglia [34] and generate ROS [35], the contribution of complement activation to $A \beta$ 's neurotoxicity (and to cognitive decline, to the extent that it is associated with plaques) is unclear. We performed regression analysis in an effort to determine the relationship between plaque-associated complement activation and cognition, but the analysis was not informative because of multicollinearity between Bielschowsky-stained and complement-stained plaques. This problem would be present regardless of the numbers of specimens in this study, because statistical power has no bearing on multicollinearity; when two variables are strongly correlated, the strength of this association is independent of the group sizes for the variables [29]. A posthoc power analysis indicated that this study was highly powered: the range of the power for detecting betweengroup differences with a 0.05 significance level, based upon the standard deviations within each of the three groups, was $78.4 \%$ to $98.3 \%$ for iC3b staining, $72.7 \%$ to $99.98 \%$ for C9 staining, and $98.8 \%$ to $99.997 \%$ for Bielschowsky staining (data not shown).

The aggregated, fibrillar $A \beta$ in neuritic plaques is considered to be the major factor through which plaques activate complement $[10,36]$, although nonfibrillar $A \beta$ in diffuse plaques may also be able to do so ([37]; however, see Nybo et al. [38]). Previous studies have detected early complement activation proteins on both diffuse and neuritic plaques $[1,2,4,12,39]$, but late-stage complement activation on plaques has been demonstrated primarily, or exclusively, on neuritic plaques [2,3,40,41]. Little or no late-stage complement activation has been reported on plaques in non-demented subjects $[3,4]$. Our results differ somewhat from these studies in that, in each of the three groups of specimens, plaque staining was detected for C9 (late-stage activation) as well as for iC3b (early-stage activation); in addition, C9 staining was occasionally observed on diffuse plaques, as well as more frequently on neuritic plaques. The slight increases in C9-stained vs. iC3b-stained plaques in MCI and AD specimens are likely to be due to differences in antibody sensitivity (i.e., the polyclonal anti-C9 may detect more plaques than monoclonal anti-iC3b). Detection of C9 on some diffuse plaques suggests that complete complement activation may, in some cases, occur on plaques even in the absence of fibrillar $A \beta$; whether this contributes to plaque "progression" from diffuse to neuritic is unknown. The similar numbers of Bielschowsky-stained and complementstained plaques in AN and MCI specimens suggest that neither increased plaque formation nor increased plaqueassociated complement activation is required for the development of early cognitive deficits. Our finding of similar plaque counts between AN and MCI subjects is in agreement with a recent study by Petersen et al. [42].

In addition to plaques, complement can be activated in the brain by NFTs [43]. NFT density and distribution are more strongly correlated than plaques with dementia severity $[44,45]$. There were few or no NFTs in most AN and MCI specimens in this study, in agreement with reports that only in severe dementia are large numbers of neocortical NFTs typically present $[46,47]$. To determine the relationship between NFT complement activation and cognition, examination of limbic structures such as the hippocampus and entorhinal cortex, in which NFT counts increase early in the disease process [47], would be required. The resulting analysis would still, most likely, be confounded by multicollinearity. Zanjani et al. [4] found little complement staining on NFTs in the entorhinal cortex except in late-stage AD, suggesting that complement activation on NFTs during the development of AD may be minimal. 
Neuronal complement staining was present in many of the specimens in this study, but there were no differences between groups for this immunoreactivity and it was poorly correlated with cognitive measures. It is unclear whether this staining indicates complement activation on neurons, neuronal synthesis of native complement proteins, or both. The monoclonal anti-iC3b antibody used in this study is stated by its manufacturer, Quidel, to be iC3b-specific, and to not recognize native $\mathrm{C} 3$. This suggests that neuronal iC3b staining is likely to represent complement activation rather than native complement protein synthesis. If neuronal C9 immunoreactivity also represents complement activation (i.e., C5b-9 deposition), then it is unclear why the MAC should be present on normal-appearing neurons. This staining could represent sublytic concentrations of C5b-9, which have been suggested to be neuroprotective [8]. Our findings do not point to complement activation as a direct cause of neuronal injury in $\mathrm{AD}$, although, as stated above, complementmediated chemotactic attraction of microglia and activation of these cells could indirectly result in neuronal damage due to microglial ROS secretion. Our detection of complement immunoreactivity on normal-appearing neurons differs from an earlier study [48] in which C1q and MAC staining was reported in the AD brain on degenerating neurons. The reasons for these conflicting results are unknown, but may be related to differences in the antibodies employed for complement staining and/or procedures for processing the brain specimens.

Our results demonstrate the limitations of studies with human brain specimens for determining the role of complement activation in AD. Although the extent of this process in the brain correlates strongly with cognitive loss, differentiating its effects from the effects of $\mathrm{AD}$ neuropathology on cognition is difficult. Studies in animal models of $\mathrm{AD}$ may eventually be of value in resolving this issue if MAC deposition can be demonstrated to the same extent in these models as in $\mathrm{AD}$. A trial in $\mathrm{AD}$ patients with an inhibitor of late-stage complement activation such as Eculizumab (Soliris, Alexion Pharmaceuticals) [49], a humanized anti-C5 monoclonal antibody which prevents formation of C5b-9, might also help to resolve this issue.

\section{Conclusion}

Early-stage (iC3b) and late-stage (C9) complement activation occurs on low numbers of plaques in the inferior temporal gyrus in both aged normal individuals and subjects with mild cognitive impairment. Complement staining on plaques is highly correlated with total plaque counts, and both of these parameters are inversely associated with measures of cognitive function. But the strong association between the numbers of complement-stained plaques and total plaque counts results in multicollinearity, which prevents a quantitative assessment of the extent to which complement activation may mediate the association of plaques with cognition during the development of $\mathrm{AD}$.

\section{Competing interests}

The author(s) declare that they have no competing interests.

\section{Authors' contributions}

DAL performed the immunocytochemical staining, plaque counts, assessment of neuronal complement immunoreactivity, and wrote the manuscript. DMC assisted in data collection (capturing of images via the image analysis system) and assessment of neuronal complement staining, generated the figures, performed some of the statistical analyses, and assisted with the writing of the manuscript. DAB assisted in experimental design, obtaining of brain specimens and clinical and post-mortem data, statistical analyses, and provided input during the drafting of the manuscript. All authors read and approved the final manuscript.

\section{Acknowledgements}

We thank Jon Wilson, M.D., Dalia Yousif, Irene Chan, Peggy Wenk, and Mamtha Balasubramamian from Beaumont Hospital, Anne Barz, Karen Skish, Julie Schneider, and Woojeong Bang from the Rush ADC, and Paul Juneau from Pfizer Global Research and Development. This study was funded by grants to D.L. from the Alzheimer's Association, and to D.B. from the NIA (P3OAGI0I6I; ROIAG 15819). Neither the Alzheimer's Association nor the NIA played a role in study design, collection, analysis and interpretation of data, manuscript preparation, or the decision to submit the manuscript for publication.

\section{References}

I. Eikelenboom P, Stam FC: Immunoglobulins and complement factors in senile plaques. An immunoperoxidase study. Acta Neuropathol (Berl) 1982, 57:239-242.

2. McGeer PL, Akiyama H, Itagaki S, McGeer EG: Activation of the classical complement pathway in brain tissue of Alzheimer patients. Neurosci Lett 1989, 1 07:341-346.

3. Webster S, Lue L-F, Brachova L, Tenner AJ, McGeer PL, Terai K, Walker DG, Bradt B, Cooper NR, Rogers J: Molecular and cellular characterization of the membrane attack complex, C5b-9, in Alzheimer's disease. Neurobiol Aging 1997, 18:415-421.

4. Zanjani H, Finch CE, Kemper C, Atkinson J, McKeel D, Morris JC, Price JL: Complement activation in very early Alzheimer disease. Alzheimer Dis Assoc Disord 2005, 19:55-66.

5. Gasque P, Neal JW, Singhrao SK, McGreal EP, Dean YD, Van BJ, Morgan BP: Roles of the complement system in human neurodegenerative disorders: pro-inflammatory and tissue remodeling activities. Mol Neurobiol 2002, 25:I-17.

6. van Beek J, Nicole O, Ali C, Ischenko A, MacKenzie ET, Buisson A, Fontaine $\mathrm{M}$ : Complement anaphylatoxin $\mathrm{C} 3 \mathrm{a}$ is selectively protective against NMDA-induced neuronal cell death. Neuroreport 200I, I 2:289-293.

7. O'Barr SA, Caguioa J, Gruol D, Perkins G, Ember JA, Hugli T, Cooper NR: Neuronal expression of a functional receptor for the $\mathbf{C 5 a}$ complement activation fragment. J Immunol 200I, 166:4154-4162.

8. Rus H, Cudrici C, Niculescu F, Shin ML: Complement activation in autoimmune demyelination: dual role in neuroinflammation and neuroprotection. J Neuroimmunol 2006, 180:9-16.

9. Shen Y, Halperin JA, Lee CM: Complement-mediated neurotoxicity is regulated by homologous restriction. Brain Res 1995, 671:282-292. 
10. Rogers J, Cooper NR, Webster S, Schultz J, McGeer PL, Styren SD, Civin WH, Brachova L, Bradt B, Ward P, Lieberburg I: Complement activation by $\beta$-amyloid in Alzheimer disease. Proc Natl Acad Sci USA 1992, 89:10016-10020.

II. Bradt BM, Kolb WP, Cooper NR: Complement-dependent proinflammatory properties of the Alzheimer's disease beta-peptide. J Exp Med 1998, I 88:43 I-438.

12. Yasojima K, Schwab C, McGeer EG, McGeer PL: Up-regulated production and activation of the complement system in Alzheimer's disease brain. Am J Pathol 1999, I 54:927-936.

13. Schultz J, Schaller J, McKinley M, Bradt B, Cooper N, May P, Rogers J: Enhanced cytotoxicity of amyloid beta-peptide by a complement dependent mechanism. Neurosci Lett 1994, I 75:99-I02.

14. Wyss-Coray T, Yan F, Lin AH, Lambris JD, Alexander JJ, Quigg RJ, Masliah E: Prominent neurodegeneration and increased plaque formation in complement-inhibited Alzheimer's mice. Proc Natl Acad Sci USA 2002, 99:10837-10842.

I5. Fonseca MI, Zhou J, Botto M, Tenner AJ: Absence of Clq leads to less neuropathology in transgenic mouse models of Alzheimer's disease. J Neurosci 2004, 24:6457-6465.

16. Schwab C, Hosokawa M, McGeer PL: Transgenic mice overexpressing amyloid beta protein are an incomplete model of Alzheimer disease. Exp Neurol 2004, I 88:52-64.

17. Webster SD, Tenner AJ, Poulos TL, Cribbs DH: The mouse CIq A-chain sequence alters beta-amyloid-induced complement activation. Neurobiol Aging 1999, 20:297-304.

18. Lue LF, Brachova L, Civin WH, Rogers J: Inflammation, A beta deposition, and neurofibrillary tangle formation as correlates of Alzheimer's disease neurodegeneration. J Neuropathol Exp Neurol 1996, 55:1083-1088.

19. Fernandez G, Heitkemper P, Grunwald T, Van Roost D, Urbach H, Pezer N, Lehnertz K, Elger CE: Inferior temporal stream for word processing with integrated mnemonic function. Hum Brain Mapp 200I, | 4:25 I-260.

20. Freedman DJ, Riesenhuber M, Poggio T, Miller EK: Experiencedependent sharpening of visual shape selectivity in inferior temporal cortex. Cereb Cortex 2006, I6: I63 I-I644.

21. Beach TG, Kuo YM, Spiegel K, Emmerling MR, Sue LI, Kokjohn K, Roher AE: The cholinergic deficit coincides with Abeta deposition at the earliest histopathologic stages of Alzheimer disease. J Neuropathol Exp Neurol 2000, 59:308-313.

22. Halliday GM, Double KL, Macdonald V, Kril J]: Identifying severely atrophic cortical subregions in Alzheimer's disease. Neurobiol Aging 2003, 24:797-806.

23. Bennett DA, Schneider JA, Aggarwal NT, Arvanitakis Z, Shah RC, Kelly JF, Fox JH, Cochran EJ, Arends D, Treinkman AD, Wilson RS: Decision rules guiding the clinical diagnosis of Alzheimer's disease in two community-based cohort studies compared to standard practice in a clinic-based cohort study. Neuroepidemiology 2006, 27:169-176.

24. Bennett DA, Wilson RS, Schneider JA, Evans DA, Aggarwal NT, Arnold SE, Cochran EJ, Berry-Kravis E, Bienias JL: Apolipoprotein E epsilon4 allele, AD pathology, and the clinical expression of Alzheimer's disease. Neurology 2003, 60:246-252.

25. Bennett DA, Schneider JA, Wilson RS, Bienias JL, Berry-Kravis E, Arnold SE: Amyloid mediates the association of apolipoprotein E e4 allele to cognitive function in older people. J Neurol Neurosurg Psychiatry 2005, 76: I I94-II99.

26. Yamamoto T, Hirano A: A comparative study of modified Bielschowsky, Bodian and thioflavin S stains on Alzheimer's neurofibrillary tangles. Neuropathol Appl Neurobiol 1986, I 2:3-9.

27. Gallyas F: Silver staining of Alzheimer's neurofibrillary changes by means of physical development. Acta Morphol Acad Sci Hung 197I, 19: |-8.

28. Loeffler DA, Camp DM, Conant SB: Complement activation in the Parkinson's disease substantia nigra: an immunocytochemical study. I Neuroinflammation 2006, 3:29.

29. Neter J, Kutner MH, Nachtsheim CJ, Wasserman W: Applied Linear Regression Models 3rd edition. Irwin/McGraw Hill Companies, Inc; 1996.

30. Webster S, Glabe C, Rogers : Multivalent binding of complement protein $\mathrm{Cl}$ q to the amyloid beta-peptide ( $\mathrm{A}$ beta) promotes the nucleation phase of $\mathbf{A}$ beta aggregation. Biochem Biophys Res Commun 1995, 2 I 7:869-875.

31. Stoltzner SE, Grenfell TJ, Mori C, Wisniewski KE, Wisniewski TM, Selkoe DJ, Lemere CA: Temporal accrual of complement pro- teins in amyloid plaques in Down's syndrome with Alzheimer's disease. Am J Pathol 2000, 156:489-499.

32. Nolte C, Moller T, Walter T, Kettenmann H: Complement 5a controls motility of murine microglial cells in vitro via activation of an inhibitory G-protein and the rearrangement of the actin cytoskeleton. Neuroscience 1996, 73: I091-II07.

33. Qin L, Liu Y, Cooper C, Liu B, Wilson B, Hong JS: Microglia enhance beta-amyloid peptide-induced toxicity in cortical and mesencephalic neurons by producing reactive oxygen species. J Neurochem 2002, 83:973-983.

34. Jekabsone A, Mander PK, Tickler A, Sharpe M, Brown GC: Fibrillar beta-amyloid peptide Abetal-40 activates microglial proliferation via stimulating TNF-alpha release and $\mathrm{H} 2 \mathrm{O} 2$ derived from NADPH oxidase: a cell culture study. J Neuroinflammation 2006, 3:24.

35. Butterfield DA, Lauderback CM: Lipid peroxidation and protein oxidation in Alzheimer's disease brain: potential causes and consequences involving amyloid beta-peptide-associated free radical oxidative stress. Free Radic Biol Med 2002, 32:1050-1060.

36. Webster S, Bradt B, Rogers J, Cooper N: Aggregation statedependent activation of the classical complement pathway by the amyloid beta peptide. I Neurochem 1997, 69:388-398.

37. Bergamaschini L, Canziani S, Bottasso B, Cugno M, Braidotti P, Agostoni A: Alzheimer's beta-amyloid peptides can activate the early components of complement classical pathway in a CIq-independent manner. Clin Exp Immunol 1999, I I 5:526-533.

38. Nybo M, Nielsen EH, Svehag SE: Complement activation by the amyloid proteins $A$ beta peptide and beta 2-microglobulin. Amyloid 1999, 6:265-272.

39. McGeer PL, Klegeris A, Walker DG, Yasuhara O, McGeer EG: Pathological proteins in senile plaques. Tohoku J Exp Med 1994, 1 74:269-277.

40. Lue L-F, Rogers ]: Full complement activation fails in diffuse plaques of the Alzheimer's disease cerebellum. Dementia 1992, 3:308-313.

41. McGeer PL, Kawamata T, Walker DG, Akiyama H, Tooyama I, McGeer EG: Microglia in degenerative neurological disease. Glia 1993, 7:84-92.

42. Petersen RC, Parisi JE, Dickson DW, Johnson KA, Knopman DS, Boeve BF, Jicha GA, Ivnik RJ, Smith GE, Tangalos EG, Braak H, Kokmen $E$ : Neuropathologic features of amnestic mild cognitive impairment. Arch Neurol 2006, 63:665-672.

43. Shen Y, Lue L, Yang L, Roher A, Kuo Y, Strohmeyer R, Goux WJ, Lee V, Johnson GV, Webster SD, Cooper NR, Bradt B, Rogers J: Complement activation by neurofibrillary tangles in Alzheimer's disease. Neurosci Lett 2001, 305:165-168.

44. Morris JC, Storandt M, McKeel DW, Rubin EH, Price JL, Grant EA Berg L: Cerebral amyloid deposition and diffuse plaques in "normal" aging: Evidence for presymptomatic and very mild Alzheimer's disease. Neurology 1996, 46:707-719.

45. Giannakopoulos P, Herrmann FR, Bussière T, Bouras C, Kövari E, Perl DP, Morrison JH, Gold G, Hof PR: Tangle and neuron numbers, but not amyloid load, predict cognitive status in Alzheimer's disease. Neurology 2003, 60: I495-1500.

46. Price JL, Davis PB, Morris JC, White DL: The distribution of tangles, plaques and related immunohistochemical markers in healthy aging and Alzheimer's disease. Neurobiol Aging 1991, 1 2:295-312.

47. Braak H, Braak E: Neuropathological stageing of Alzheimerrelated changes. Acta Neuropathol (Berl) 1991, 82:239-259.

48. D'Andrea MR: Evidence that immunoglobulin-positive neurons in Alzheimer's disease are dying via the classical antibody-dependent complement pathway. Am J Alzheimer's Dis Other Demen 2005, 20: I44-150.

49. Rother RP, Rollins SA, Mojcik CF, Brodsky RA, Bell L: Discovery and development of the complement inhibitor eculizumab for the treatment of paroxysmal nocturnal hemoglobinuria. Nat Biotechnol 2007, 25:1256-1264. 